\title{
Effectiveness and safety of bifidobacteria and berberine in people with hyperglycemia: study protocol for a randomized controlled trial
}

\author{
Jie Ming, Shaoyong Xu, Chun Liu, Xiangyang Liu, Aihua Jia and Qiuhe Ji
}

\begin{abstract}
Background: Berberine is one of the most important examples of a Chinese traditional medicine that has hypoglycemic effects but there have been no randomized controlled trials of the drug in a larger sample. In addition, the use of probiotic biotherapy to maintain an appropriate intestinal flora may represent an effective early intervention for hyperglycemia. Unfortunately, there has been a shortage of relevant research on this possibility at the population level. This study was designed to determine the hypoglycemic effect and safety of both bifidobacteria and berberine administration to newly diagnosed patients with pre-diabetes or diabetes mellitus.
\end{abstract}

Methods/design: This is a multicenter, double-blind, randomized, and parallel-controlled study that includes a run-in period of 2 weeks and a treatment period of 16 weeks, which will be conducted between June 2015 and October 2018. The 300 randomized patients will be assigned to the following four groups for 16 weeks' treatment: Bifidobacterium, berberine, Bifidobacterium combined berberine, and placebo control groups. The primary outcome is the absolute value of fasting plasma glucose compared with baseline after 16 weeks of treatment.

Discussion: This is the first randomized controlled trial to determine the hypoglycemic effect and safety of both bifidobacteria and berberine administration to newly diagnosed patients with pre-diabetes or diabetes mellitus. It may provide support for the use of berberine and bifidobacteria in the treatment of diabetes.

Trial registration: ClinicalTrials.gov, ID: NCT03330184. Retrospectively registered on 18 October 2017.

Keywords: Bifidobacteria, Berberine, Hyperglycemia, Diabetes, Pre-diabetes, Chinese population, Randomized trials

\section{Background}

According to the latest national epidemiological survey of diabetes, China has prevalence rates of $9.7 \%$ and $15.5 \%$ for diabetes and pre-diabetes, respectively, among people of over 20 years of age [1], and it has become one of the countries with the largest number of diabetic patients. Oral hypoglycemic drugs have been widely used in China as the recommended first-line treatment, and these can effectively reduce blood glucose [2]. However, adverse reactions or side effects can be associated with their use, albeit benefits that outweigh the risks. For example, metformin can have gastrointestinal effects,

\footnotetext{
* Correspondence: jqiuhe@fmmu.edu.cn

Department of Endocrinology, Xijing Hospital, Fourth Military Medical

University, 169 Changle Road West, Xi'an 710032, China
}

sulfonylureas can cause hypoglycemia, and glucagon-like peptide- 1 analogs can induce gastrointestinal reactions and antibody formation [3, 4]. Although insulin is relative effective and safe, it may also have risks of hypoglycemia, must be administered by injection and, therefore, its use is less straightforward. Therefore, it is necessary to develop novel effective oral hypoglycemic agents.

Chinese traditional medicine has been used for thousands of years. Diabetes and its treatment were described as early as the Wei and Jin periods (A.D. 220-420), and "Huanglian treats diabetes" was published in the Renown Physicians' Extra Records [5]. With the modernization of traditional Chinese medicine, its use for the treatment of diabetes has attracted greater attention from researchers in recent years. Berberine is one of the most important 
examples of a traditional Chinese medicine that has hypoglycemic effects [6]. It has been used for the treatment of the complications of diabetes in China for 1500 years $[7,8]$. Some studies reported that berberine can improve blood glucose and lipid levels in patients with type 2 diabetes mellitus and dyslipidemia [9-11]. A possible mechanism for its beneficial effects is an increase in the expression and activity of glucose transporter 1, thus increasing the glucose consumption of cells in a non-insulin dependent manner. In addition, it may reversibly inhibit insulin expression, as well as increasing insulin sensitivity at the level of the insulin receptor and activating the insulin signal transduction pathway [12-16].

In addition, because the intestinal tract represents the first organ to be exposed to glucose, and, therefore, has a key role in glucose metabolism, many researchers now believe that the composition of the intestinal flora is important in metabolic diseases such as type 2 diabetes [17-19]. An imbalance in the intestinal flora can result in the presence of deleterious metabolites in the body and cause metabolic endotoxemia, leading to an inflammatory response, the development of insulin resistance, and eventually type 2 diabetes [20]. However, probiotics, such as bifidobacteria, are protective against diabetes because they maintain balance in the intestinal flora $[21,22]$. In addition, they may indirectly impact glucose metabolism through effects on intestinal hormones and/ or the exocrine pancreas. Previous studies have shown that supplementation with bifidobacteria can improve glucose tolerance and glucose-induced insulin secretion by reducing endotoxemia and proinflammatory cytokine secretion by adipose tissue in high-fat-diet-induced diabetic mice, thus reducing inflammation and ameliorating the metabolic disorders associated with diabetes [22]. Therefore, the use of probiotic biotherapy to maintain an appropriate intestinal flora may represent an effective early intervention for diabetes. Unfortunately, there has been a shortage of relevant research on this possibility at the population level.

Thus, this study was designed to determine the hypoglycemic effect and safety of both bifidobacteria and berberine administration to newly diagnosed patients with pre-diabetes or diabetes mellitus, and their impact on the cardiovascular system, blood lipid metabolism, the intestinal flora, intestinal hormones, body weight, and quality of life.

\section{Methods/design}

This is a multicenter, double-blind, randomized, and parallel-controlled study that includes a run-in period of 2 weeks and a treatment period of 16 weeks, which will be conducted between June 2015 and October 2018.

\section{Institutional setting}

This multicenter study will be conducted in eight research centers in Xi'an, Shaanxi Province, China: the First Affiliated Hospital of the Fourth Military Medical University, the First Affiliated Hospital of Xi'an Jiaotong University, the Second Affiliated Hospital of Xi'an Jiaotong University, Shaanxi People's Hospital, Xi'an Chang'an Hospital, Xi'an High-tech Hospital, Xi'an Central Hospital, and Shaanxi Aerospace Hospital.

\section{Research population and inclusion criteria}

The inclusion and exclusion criteria are shown in the Appendix.

\section{Pharmacologic treatments}

The study will include an introductory period of 2 weeks and treatment for 16 weeks (Fig. 1). After the introductory period, the 300 successfully screened subjects will undergo baseline data acquisition and lifestyle guidance and be randomly assigned to the following four groups for 16 weeks' treatment: Bifidobacterium, berberine, combination, and control groups.

The berberine group will receive berberine tablets, $0.5 \mathrm{~g}$, two a day, and Bifidobacterium placebo capsules, $0.70 \mathrm{~g}$, two a day, orally. The Bifidobacterium group will receive Bifidobacterium viable capsules, $0.70 \mathrm{~g}$, two a day, and berberine placebo tablets, $0.5 \mathrm{~g}$, two a day, orally. The combination group will receive Bifidobacterium viable capsules, $0.70 \mathrm{~g}$, two a day, and berberine tablets, $0.5 \mathrm{~g}$, two a day, orally. The control group will receive Bifidobacterium placebo capsules, $0.70 \mathrm{~g}$, two a day, and berberine placebo tablets, $0.5 \mathrm{~g}$, two a day, orally.

The preparations used will be berberine tablets (Northeast Pharmaceutical Group Shenyang First Pharmaceutical Co., Ltd., Lot No.: 130917), berberine tablet simulant (Northeast Pharmaceutical Group Shenyang First Pharmaceutical Co., Ltd., Lot No.: 20150101), Bifidobacterium viable capsules (Livzon Pharmaceutical Group, Lot No.: 20141013), and Bifidobacterium viable capsules simulant (Livzon Pharmaceutical Group, Lot No.: 141001).

During the study, researchers will administer other drugs that are not defined within the exclusion criteria and are necessary for the safety and health of the subjects to treat disease or discomfort. All of the drugs administered during the study will be recorded in the corresponding parts of the appropriate Case Report Form (CRF). Here, the specific type of drug (trade name or common name), indications for use, the date, and the dose used will be recorded. In addition, the following drugs will be banned or restricted during the study: any hypoglycemic therapy except for the study drugs, and 


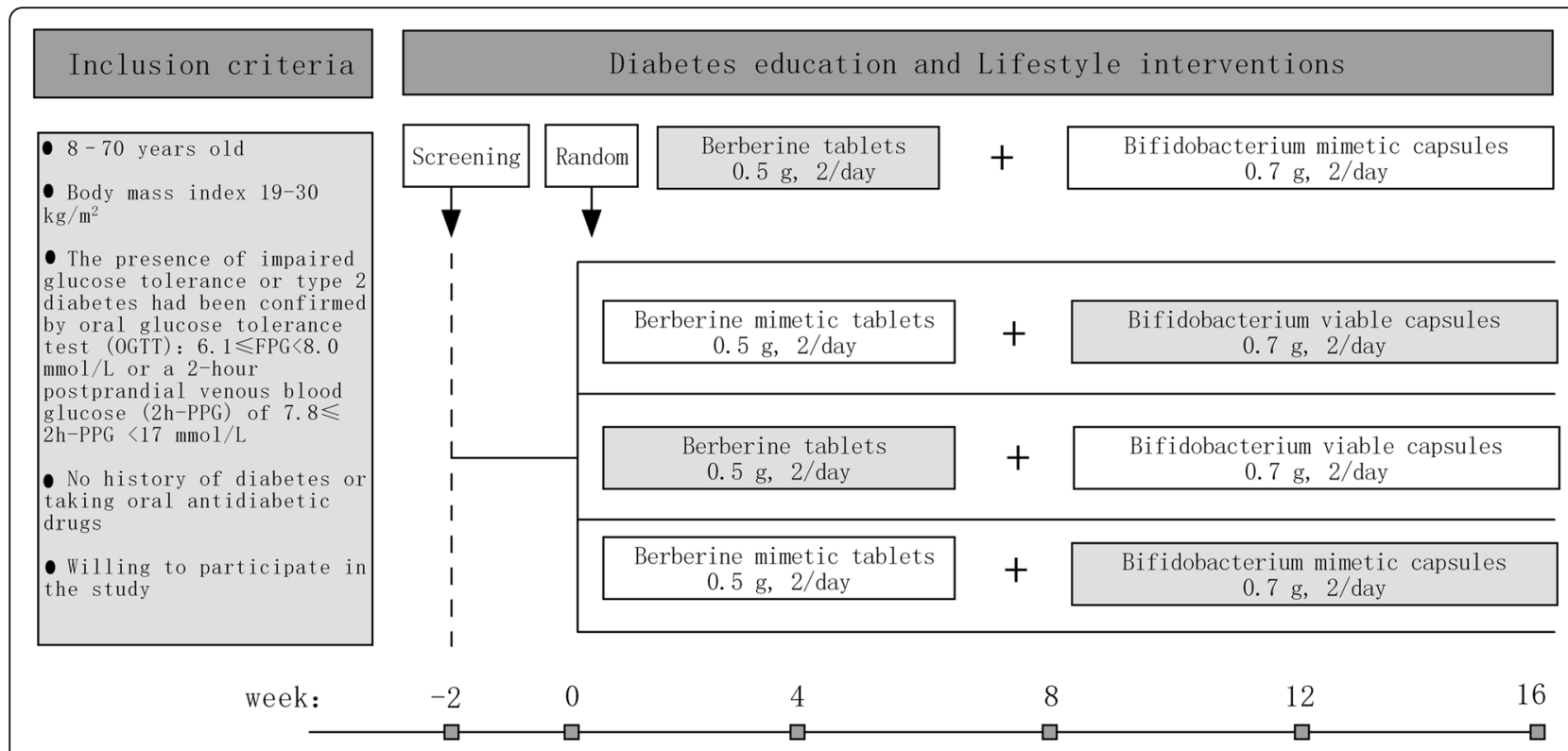

Fig. 1 Inclusion criteria and interventions

systemic glucocorticoid (orally or intravenously administered), if used for more than seven consecutive days.

\section{Lifestyle interventions}

Regulation of the diet and exercise of the subjects will be regarded as very important in this study, and discussion and implementation of a diet and exercise plan will be commenced in accordance with researchers' suggestions from the beginning of the screening period to the end of the trial. A standardized meal suggestion (30 $\mathrm{kcal} / \mathrm{kg}$ ideal weight/day, based on dietitian recommendations), will be provided, which includes $55 \%$ of calories from carbohydrate, $25 \%$ from fat, and 20\% from protein, with these macronutrients being present in the respective proportions: $1 / 5,2 / 5$, and $2 / 5$, and participants will be asked to be strict about their diet and to avoid short-term or high-intensity exercise. Researchers will emphasize the importance of diet and exercise to subjects at each treatment visit.

\section{Outcomes}

The main and secondary outcomes are shown in Table 1.

\section{Participant timeline}

The time course for participant registration, intervention, assessment, and follow-up is shown in the Standard Protocol Items: Recommendations for Interventional Trials (SPIRIT) Figure (Fig. 2).

\section{Randomization}

The stratified blocked randomization method will be used. Stratification will be conducted by each research

Table 1 Primary and secondary outcomes of the trial

\begin{tabular}{|c|c|c|}
\hline Purpose & & Measurements \\
\hline $\begin{array}{l}\text { Primary } \\
\text { outcome }\end{array}$ & & The absolute value of fasting plasma glucose (FPG) compared with baseline after 16 weeks of treatment \\
\hline $\begin{array}{l}\text { Secondary } \\
\text { outcomes }\end{array}$ & 1 & $\begin{array}{l}\text { The proportion of patients who meet following blood glucose response: (1) HbA1c }<7.0 \% \text {; (2) HbA1c }<7.0 \% \text {, without severe } \\
\text { hypoglycemia; (3) HbA1c }<7.0 \% \text {, without diagnosed hypoglycemia; (4) HbA1c }<6.0 \% \text {; (5) HbA1c }<6.0 \% \text {, without severe } \\
\text { hypoglycemia; (6) HbA1c }<6.0 \% \text {, without diagnosed hypoglycemia; (7) FPG }<110 \mathrm{mg} / \mathrm{dL}(6.1 \mathrm{mmol} / \mathrm{L}) \text {; (8) FPG }<130 \mathrm{mg} / \mathrm{dL}(7.2 \mathrm{mmol} / \mathrm{L})\end{array}$ \\
\hline & 2 & $\begin{array}{l}\text { Changes compared with baseline: (1) 2-h postprandial glucose (2-h PPG): (2) glycosylated hemoglobin (HbA1c); (3) } 2 \text { h PPG < } 140 \text { mg/dL } \\
\text { (7.8 mmol/L); (4) 2-h PPG <180 mg/dL (10.0 mmol/L); (5) HbA1c reduced by 0.5\%; (6) blood pressure (BP); (7) lipid changes: total } \\
\text { cholesterol (Total-C), low-density lipoprotein cholesterol (LDL-C), high-density lipoprotein cholesterol (HDL-C), and triglyceride (TG); } \\
\text { (8) Body weight and Body Mass Index (BMl); (9) HOMA Index, insulin early phase and late-phase secretion index; (10) intestinal hormones } \\
\text { (GLP-1, glucagon); (11) intestinal flora }\end{array}$ \\
\hline
\end{tabular}

Safety Laboratory data: electrocardiograph (ECG); pulse and blood pressure (BP); weight and physical examination. 


\begin{tabular}{|c|c|c|c|c|c|c|c|}
\hline \multirow{2}{*}{\begin{tabular}{|l|} 
Research stages \\
Visiting No. (Office) \\
\end{tabular}} & \multirow{2}{*}{$\begin{array}{c}\begin{array}{c}\text { Screening } \\
\text { period }\end{array} \\
\text { V1 }\end{array}$} & \multicolumn{6}{|c|}{ Treatment period } \\
\hline & & \begin{tabular}{|c|} 
V2 \\
Randomization
\end{tabular} & Tel Visit & v3 & v4 & v5 & v6 \\
\hline Research cycle (visiting time window) & $-2 w \pm 7 d$ & Ow Baseline & $2 w \pm 7 d$ & $4 w \pm 7 d$ & $8 w \pm 7 d$ & $12 w \pm 7 d$ & $16 w \pm 7 d$ \\
\hline \multicolumn{8}{|l|}{ Demographic data/baseline } \\
\hline Informed consent & $v$ & & & & & & \\
\hline Inclusion/exclusion criteria & $v$ & $v$ & & & & & \\
\hline Demographic data & $v$ & & & & & & \\
\hline Physical examination & $v$ & $v$ & & $\checkmark$ & $v$ & $v$ & $r$ \\
\hline Vital signs & $v$ & $v$ & & $v$ & $v$ & $v$ & 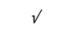 \\
\hline Complications \& combined medication & $\mathrm{v}$ & v & $\checkmark$ & $v$ & $v$ & $v$ & v \\
\hline Lifestyle guidance & $v$ & $v$ & $v$ & $v$ & $v$ & $v$ & $v$ \\
\hline \multicolumn{8}{|l|}{ Treatment period } \\
\hline Diet survey & & $v$ & & $v$ & v & $v$ & $r$ \\
\hline Blood glucose meter distribution & & v & & & & & \\
\hline Research diary release and recycling & & $v$ & & $v$ & $v$ & $v$ & $\mathrm{r}$ \\
\hline Study drug distribution & & v & & $v$ & $\mathrm{v}$ & $\mathrm{v}$ & \\
\hline Study drug recycling and count & & . & & $v$ & $\mathrm{v}$ & $v$ & 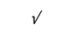 \\
\hline Associated drug & & $v$ & & $v$ & $v$ & $v$ & $r$ \\
\hline \multicolumn{8}{|l|}{ Safety assessment } \\
\hline Pregnancy test (urine) & $v$ & $v$ & & $v$ & $v$ & $v$ & $\mathrm{v}$ \\
\hline Physical examination & $v$ & $v$ & & $v$ & $v$ & $v$ & $v$ \\
\hline Vital signs & $v$ & $v$ & & $v$ & v & $v$ & $v$ \\
\hline 12 lead electrocardiograms & $v$ & $v$ & & $v$ & & & $v$ \\
\hline Blood and urine routine measurements & $v$ & $v$ & & $v$ & & & $v$ \\
\hline Liver function & $v$ & v & & $v$ & & & $v$ \\
\hline Renal function & $v$ & $v$ & & $v$ & & & v \\
\hline Blood lipid test & $v$ & $v$ & & $v$ & & & $v$ \\
\hline Adverse events & $v$ & $v$ & $v$ & $v$ & v & $v$ & $\mathrm{v}$ \\
\hline \multicolumn{8}{|l|}{ Validity assessment } \\
\hline Fasting plasma glucose & $v$ & $v$ & & $v$ & $\checkmark$ & $\checkmark$ & $v$ \\
\hline Glycosylated hemoglobin & & $v$ & & & & & $v$ \\
\hline OGTT test (BG, Insulin) & & $v$ & & & & & $v$ \\
\hline SMBG & & 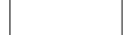 & & $v$ & v & $v$ & $v$ \\
\hline Body weight and BMI & $v$ & $v$ & & $v$ & $v$ & $v$ & $v$ \\
\hline \multicolumn{8}{|l|}{ Other assessments } \\
\hline Intestinal flora & & $v$ & & & & & v \\
\hline Intestinal hormones $(0,30 \mathrm{~min}, 2 \mathrm{~h})$ & & $v$ & & & & & $v$ \\
\hline \multicolumn{8}{|l|}{ Backup specimens } \\
\hline Stool specimens & & $v$ & & & & & v \\
\hline Serum specimens & & $v$ & & & & & $v$ \\
\hline Anticoagulant specimens & & $v$ & & & & & $v$ \\
\hline
\end{tabular}

Fig. 2 Standard Protocol Items: Recommendations for Interventional Trials (SPIRIT) Figure. Specific research plan and implementation steps. Note: Laboratory safety assessment assays were completed by sub-center laboratories, while the validity assessment laboratory assays and the intestinal indices were completed by the central laboratory. OGTT oral glucose tolerance test, BG blood glucose, SMBG self-monitoring of blood glucose, BMI Body Mass Index

center to select the appropriate segment length. The statistical software SAS 8.2 PROC PLAN will be used to generate a random code list for 300 subjects to receive specific treatments. All randomized grouping number segments will be sent to the research centers with the corresponding treatment kits. Researchers will then select kits with the same series number for the study according to the visit sequence. 


\section{Blinding and unblinding procedures}

Because the two research drugs have different specifications and appearance, double-blind dual simulation will be selected as the method of blinding. The test and placebo drugs will be provided by the coordinating center in identical internal and external packaging.

A two-level blinding design will be used, with the first level being by group (groups $\mathrm{A}, \mathrm{B}, \mathrm{C}$, and $\mathrm{D}$ ) according to case number, and the second level by treatment (berberine, Bifidobacterium, combination and control). The random code list will be created by a statistical agency, the two-level treatment codes will be sealed separately in duplicate, with a copy being kept by each of the statistical agency and the central coordinator, and the treatment code will be not opened during the study. The study would have been considered invalid if any treatment code disclosure arose from any nonprescribed circumstances and if it affected the objectivity of the data obtained from the study.

A two-phase unblinding method will be adopted. When all of the CRFs have been entered into the database, the first unblinding (identifying groups A, B, C, and D) will be carried out after the blind review, data locking, and statistical analysis plans will be confirmed. The second unblinding will be conducted when the statistical analysis report is completed, to identify subjects in the berberine, Bifidobacterium, combination and control groups.

Each coded test drug will have a corresponding emergency letter, describing the subject's medication, treatment methods, and the organization to which requests for emergency unblinding should be immediately reported. Emergency letters will be sealed and issued to the research center with the corresponding numbers, where they are preserved and not opened unless necessary. In the case of an emergency (for example, a severe adverse reaction), when the type of medication being administered must be known for the effective treatment of the subject, the researcher could access the appropriate letter. In this instance the identity of the opener, the opening date, and the reasons for doing so will be recorded on the CRF. Once unblinded, the subjects will withdraw from the trial, and be treated as expulsion cases. All emergency letters and CRFs will be collected at end of the trial for blind review.

\section{Data collection}

Laboratory parameters will be used for validity and safety assessments (see Fig. 2 for details). The central laboratory will provide all the appropriate materials needed by the research centers for sample collection, processing, packaging, and transportation. Before starting the clinical study, a laboratory manual will be provided to center researchers, containing a detailed description. Insulin, glucagon, and GLP-1 will be measured by the central laboratory, while other assays are carried out by the sub-centers, according to the unified standard provided by the coordinator.

Before blood collection, subjects will sit for $5 \mathrm{~min}$. A tourniquet will be used for up to $2 \mathrm{~min}$. For serum insulin assay, $5 \mathrm{ml}$ whole blood will be taken while fasting, and $30 \mathrm{~min}$ and $2 \mathrm{~h}$ after glucose intake. This will be centrifuged for $2 \mathrm{~h}$, and the separated serum will be collected into cryopreservation tubes. For glycosylated hemoglobin (HbA1c) measurement $2 \mathrm{ml}$ whole blood will be obtained and placed into blood collection tubes containing EDTA. For GLP-1 and glucagon assay, $5 \mathrm{ml}$ blood will be obtained using vacuum blood collection tubes containing EDTA and aprotinin, after fasting, and $30 \mathrm{~min}$ and $2 \mathrm{~h}$ after a meal, and the centrifuged serum will be collected into cryopreserved tubes for storage at $-20{ }^{\circ} \mathrm{C}$. Stool specimens the size of soybeans will be placed into dry, sterile collection tubes, and sent to the analysis center within $24 \mathrm{~h}$ for cryopreservation.

Prior to undergoing an oral glucose tolerance test (OGTT) subjects will consume a normal diet (with carbohydrate content no less than $150 \mathrm{~g}$ ) for at least 3 days. After 10-12 $\mathrm{h}$ of fasting, they will be required to empty their bladder and an initial blood sample will be obtained. Then they will drink $75 \mathrm{~g}$ anhydrous glucose dissolved in $300 \mathrm{ml}$ water over approximately $5 \mathrm{~min}$, and the time of ingestion will be recorded. Further blood samples will be then obtained after $30 \mathrm{~min}$ and $2 \mathrm{~h}$.

\section{Statistical methods}

Because no previous studies of treatment with bifidobacteria have been conducted, this represents a pilot study. Sample size was estimated using NCSS PASS 11 (NCSS LLC, Kaysville, UT, USA) software, and it was planned that 300 subjects would be enrolled. The detailed statistical methods are described below.

Superiority hypothesis: in case of occurrence of superiority of either the berberine, Bifidobacterium or combination group, the test will be deemed successful, with the bilateral test level for $\alpha=5 \%$ (no correction), and for $\beta=20 \%$ (power $80 \%$ ).

The primary observation indicator in this study was the absolute value of fasting plasma glucose (FPG) compared with baseline after 16 weeks of treatment. The berberine sample size was estimated with reference to the STOP-NIDDM study [4] and Yifei Zhang's study [11] on the use of berberine to treat type 2 diabetes with hyperlipidemia. In this study, berberine treatment decreased FPG by $1.45 \pm 0.85$ (standard deviation (SD)) $\mathrm{mmol} / \mathrm{L}$, while FPG in the placebo control group decreased by $0.6 \pm 1.2 \mathrm{mmol} / \mathrm{L}$. With a difference in FPG between the berberine and placebo groups of 
$0.85 \mathrm{mmol} / \mathrm{L}$, the estimated pooled variance was 2 , and the estimated sample size was 44 .

The bifidobacteria sample size was estimated based on a decrease in FPG of $1.2 \pm 1.1 \mathrm{mmol} / \mathrm{L}$, and a decrease in FPG in the placebo control group of $0.6 \pm 1.0 \mathrm{mmol} / \mathrm{L}$. Assuming that the difference in FPG decrease between the berberine and placebo groups was $0.6 \mathrm{mmol} / \mathrm{L}$, and the estimated pooled variance was 2, the estimated sample size was 88 persons in a single group. Allowing for some expulsions, the final sample size for each group was determined to be 50 for the berberine group, 100 for the Bifidobacterium group, 50 for the combination group, and 100 for the placebo control group, giving a total sample size of 300 .

The primary analysis population will undergo at least one trial drug treatment and have at least one followup record comprising a full analysis set (FAS) and will be analyzed on an intention-to-treat (ITT) basis, including all randomized patients. All support analyses will be based on per-protocol sets (PPSs), and the security endpoint analysis will be based on a safety set (SS). The study will not incorporate a planned midpoint analysis.

All data will be analyzed using SAS 9.3 (SAS institute Inc, Cary, NC, USA) software using pre-programmed algorithms. Quantitative indices recorded will include the mean $\pm \mathrm{SD}$, median, maximum and minimum values; qualitative or grade indices will be recorded using a frequency distribution table. Two-sided tests will be used in all cases, and $P \leq 0.05$ will be considered to be statistically significant. Fisher's exact probability test will be used to compare the expulsion rates between the groups.

Validity analysis will be based on the FAS, using a covariance analysis model, with the main and secondary efficacy index baseline values being the covariates in the model, and the central effect serving as the fixed-effect term. The least squares estimates will be used to calculate the corrected mean and the 95\% confidence intervals for changes in each group, and the $95 \%$ confidence interval of the Dunnett corrected mean difference between the test and placebo groups will be used to fit a covariance analysis model with central and group interactions to test the consistency among study centers. The applicability of this covariance analysis model will be investigated at the level of $\alpha=0.10$. Unless otherwise specified, the method of Carrying Forward (last observation carried forward, LOCF) will be used for sensitivity analysis of each validity analysis.

Security analysis will be based on a SS. Safety endpoints will be analyzed and adverse events aggregated. The continuous variables or frequency counts and percentage in safety and tolerability endpoints will be analyzed using descriptive statistics. The relative indices for hypoglycemic events and changes in weight will be compared between groups according to general principles.

\section{Discussion}

In recent years, many researchers have continued to explore applications of traditional Chinese medicine and the regulation of the intestinal flora for the treatment of diabetes. Berberine and bifidobacteria are important examples of Chinese traditional medicine and probiotics, but a well-designed, randomized, double-blind clinical trial has not been conducted to date with sufficient sample size to validate these pre-clinical findings.

Many pre-clinical studies have shown that berberine acts at the cellular metabolic level, especially in hepatocytes, pancreatic beta cells, and adipocytes. Regulation of AMP-dependent protein kinase (AMPK) plays a central role in berberine's effect on cellular pathways. A study found that berberine can increase insulin secretion and reduce antioxidant stress through this pathway [23], while another study showed that berberine can increase insulin receptor (InsR) expression, which it was shown to do in a time and dose-dependent manner in human hepatocyte cells. Berberine administration in the presence or absence of calcium phosphate protein $\mathrm{C}$ (a protein kinase C (PKC) inhibitor) or U0126 (an extracellular signal-regulated kinase inhibitor) and others demonstrated that berberine activates the InsR gene promoter mainly through activation of the PKC pathway and thus promotes expression of the InsR gene [24].

Clinical studies have also suggested that berberine is an effective therapy for diabetes and metabolic syndrome. The study of 31 cases by Yin et al. in 2008 found that berberine can reduce fasting and postprandial blood glucose by $>30 \%$ [25]. In the meta-analysis conducted by Dong et al. in 2012, 138 patients taking berberine (0.5-1.5 g/day, 8-12 weeks) and 133 taking placebo were included, and the results showed that berberine can reduce fasting plasma glucose by $12 \%$, postprandial glucose by $16 \%$, and glycosylated hemoglobin by $0.9 \%$ [26]. However, the sample sizes of the independent studies were small.

The intestinal flora has been proposed to be a human "microbial organ," which has a number of important roles in the body. Bifidobacteria are symbionts that play an important role in the regulation of the intestinal flora. Cani et al. showed that bifidobacteria improve glucose tolerance and glucose-induced insulin secretion, and reduce high-fat-diet-induced endotoxemia and serum proinflammatory cytokine levels in diabetic mice, thereby ameliorating inflammation in the mice and improving their metabolic status [22]. However, the exact relationship between intestinal bifidobacteria and type 2 diabetes is not clear. There have been fewer studies on the differences in intestinal tract bifidobacteria between type 2 
diabetes patients and healthy individuals, with no robust evidence to support a difference in the population.

This is the first large-scale, multicenter, double-blind, randomized, parallel-controlled study on berberine and bifidobacteria in China, with the objective of quantifying their hypoglycemic effects and safety in newly diagnosed cases of pre-diabetes or diabetes mellitus. It may provide support for the use of berberine and bifidobacteria in the treatment of diabetes.

\section{Trial status}

The trial is currently recruiting participants. The recruitment began in June 2015 and is anticipated to end in October 2018. Trial registration number: NCT03330184. Registration date: 18 October 2017 - retrospectively registered. Registered at ClinicalTrials.gov; the URL of the trial registry record is https://register.clinicaltrials.gov/. The trials protocol was reported according to the SPIRIT statement (Additional file 1).

\section{Appendix}

\section{Inclusion criteria}

1. Age between 17 and 70 years

2. Body Mass Index of $19-30 \mathrm{~kg} / \mathrm{m}^{2}$

3. The presence of impaired glucose tolerance or type 2 diabetes had been confirmed by oral glucose tolerance test. (The V1 stage is defined by fasting plasma glucose (FPG): $5.6 \leq \mathrm{FPG}<8.0 \mathrm{mmol} / \mathrm{L}$, while the $\mathrm{V} 2$ stage is defined by $6.1 \leq \mathrm{FPG}<8.0 \mathrm{mmol} / \mathrm{L}$ or a 2 -h postprandial venous blood glucose (2-h PPG) of $7.8 \leq 2$-h PPG $<17 \mathrm{mmol} / \mathrm{L}$ (each sub-center laboratory))

4. Having not participated in any other clinical trial within the prior 3 months

5. Having voluntarily given their informed consent

\section{Exclusion criteria}

1. Type 1 diabetes mellitus

2. Diabetes patients with previously treated or untreated $\mathrm{FBG} \geq 8 \mathrm{mmol} / \mathrm{L}$ or $2-\mathrm{h} \mathrm{PPG} \geq 17 \mathrm{mmol} / \mathrm{L}$

3. Women of childbearing potential who are pregnant, breastfeeding or intend to become pregnant or are not using adequate contraceptive methods

4. Impaired liver function, defined as aspartate aminotransferase (AST) or alanine transaminase (ALT) more than twice the upper limit of normal

5. Impaired renal function, defined as serum creatinine $\geq 133 \mu \mathrm{mol} / \mathrm{L}$

6. Uncontrolled treated/untreated severe hypertension (systolic blood pressure $\geq 160 \mathrm{mmHg}$ and/or diastolic blood pressure $\geq 95 \mathrm{mmHg}$ )

7. Chronic gastrointestinal diseases
8. Cancer and medical history of cancer (except basal cell skin cancer or squamous cell skin cancer)

9. Any clinically significant disease or disorder which, in the investigator' opinion could interfere with the results of the trial

10.Mental incapacity, psychiatric disorder, unwillingness or language barriers precluding adequate understanding or co-operation, including subjects being unable to read and write

11.Known or suspected hypersensitivity to trial products or related products

12.Known or suspected abuse of alcohol, narcotics or illicit drugs

\section{Additional file}

Additional file 1: SPIRIT 2013 Checklist: recommended items to address in a clinical trial protocol and related documents*. (DOCX $591 \mathrm{~kb}$ )

Abbreviations

AMPK: AMP-dependent protein kinase; ARF: Case Report Form; FAS: Full analysis set; FPG: Fasting plasma glucose; GLP-1: Glucagon-like peptide; InsR: Insulin receptor; ITT: Intention-to-treat; LOCF: Last observation carried forward; OGTT: Oral glucose tolerance test; PKC: Protein kinase C; PPS: Perprotocol set; SD: Standard deviation; SS: Safety analysis set

\section{Acknowledgements}

We thank Mark Cleasby, PhD, from Liwen Bianji, Edanz Group China (www.liwenbianji.cn/ac), for editing the English text of a draft of this manuscript.

\section{Funding}

This study was supported by the Foundation of Shaanxi Province, China. QJ was supported by the Natural Science Foundation of Shaanxi Province, China (Grant No. 2013KTZB03-02-01)

\section{Authors' contributions}

JM and SX contributed equally to this work. QJ and SX were involved in study concept and design. JM and $C L$ wrote the first draft; $S X, X L, A J$, and QJ contributed to the revision of the final report. All authors read and approved the final manuscript.

\section{Ethics approval and consent to participate}

This study was approved by the independent Ethics Committee of the First Affiliated Hospital of the Fourth Military Medical University (ID: KY201506113) and registered (registration date: 18 October 2017 - retrospectively registered) at the National Institutes of Health Clinical Research Trials Register (ClinicalTrials.gov, identifier: NCT03330184). The findings of this trial will be submitted to a peer-reviewed journal. Abstracts will be submitted to relevant national and international conferences.

Each subject has provided signed and dated informed consent before conducting any procedure associated with the study. Participation in this study is strictly confidential. Any information that is published will not reveal the identity of the subjects.

Competing interests

The authors declare that they have no competing interests.

\section{Publisher's Note}

Springer Nature remains neutral with regard to jurisdictional claims in published maps and institutional affiliations. 
Received: 18 November 2017 Accepted: 2 January 2018

Published online: 26 January 2018

\section{References}

1. Yang W, Lu J, Weng J, Jia W, Ji L, Xiao J, et al. Prevalence of diabetes among men and women in China. N Engl J Med. 2010;362:1090-101.

2. El-Kaissi S, Sherbeeni S. Pharmacological management of type 2 diabetes mellitus: an update. Curr Diabetes Rev. 2011;7:392.

3. Goldberg RB, Temprosa M, Haffner S, Orchard TJ, Rater RE, Fowler SE, et al. Effect of progression from impaired glucose tolerance to diabetes on cardiovascular risk factors and its amelioration by lifestyle and metformin intervention: the Diabetes Prevention Program randomized trial by the Diabetes Prevention Program Research Group. Diabetes Care. 2009:32:726-32.

4. Tuomilehto J, Lindstrom J, Hellmich M, Lehmacher W, Westermeier T, et al. Development and validation of a risk-score model for subjects with impaired glucose tolerance for the assessment of the risk of type 2 diabetes mellitus-The STOP-NIDDM risk-score. Diabetes Res Clin Pract. 2010;87:267-74.

5. Zhang J, Tang H. Berberine progress in clinical application. Pharm Clin Res. 2015:3:287-90

6. Zhang L, Ji Y. Application of berberine in diabetes treatment. J Pract Med. 1999;13:22.

7. Kong W, Wei J, Abidi $P$, Lin M, Inaba S, Li C, et al. Berberine is a novel cholesterol-lowering drug working through a unique mechanism distinct from statins. Nat Med. 2004;10:1344-51.

8. Lee YS, Kin KH, Yoon MJ, Cho HJ, Shen Y, Ye JM, et al. Berberine, a natural plant product, activates AMP-activated protein kinase with beneficial metabolic effects in diabetic and insulin-resistant states. Diabetes. 2006;55:2256-64.

9. Kupeli E, Kosar M, Yesilada E, Husnu K, Baser C. A comparative study on the anti-inflammatory, antinociceptive and antipyretic effects of isoquinoline alkaloids from the roots of Turkish Berberis species. Life Sci. 2002;72(6):645-57.

10. Tang LQ, Wei W, Chen LM, Liu S. Effects of berberine on diabetes induced by alloxan and a high-fat/high-cholesterol diet in rats. J Ethnopharmacol. 2006:108:109-15.

11. Zhang Y, Li X, Zou D, Liu W, Yang J, Zhu N, et al. Treatment of type 2 diabetes and dyslipidemia with the natural plant alkaloid berberine. J Clin Endocrinol Metab. 2008;93:2559-65

12. Yin J, Hu R, Chen M, Tang J, Li F, Yang Y, et al. Effects of berberine on glucose metabolism in vitro. Metabolism. 2002;51:1439.

13. Zhou $\mathrm{L}$, Chen $\mathrm{M}$, Song $\mathrm{H}$. Effect of berberine on glucose transfer in fat cells and its mechanism. J Chin Endocr Metab. 2003;19:479-82.

14. Zhou L, Yang Y. Effect of berberine on the glucose metabolism of fat cells. J Shanghai First Med Univ. 2002;22:412-4.

15. Zhou L, Yang Y, Wang X, Liu S, Shang W, Yuan G, et al. Berberine stimulates glucose transport through a mechanism distinct from insulin. Metabolism. 2007:56:405-12

16. Bustanji Y, Taha MO, Yousef AM, Al-Bakri AG. Berberine potently inhibits protein tyrosine phosphatase 1B: investigation by docking simulation and experimental validation. J Enzyme Inhib Med Chem. 2006;21:163-71.

17. Cani PD, Delzenne NM. Gut microflora as a target for energy and metabolic homeostasis. Curr Opin Clin Nutr Metab Care. 2007:10:729-34.

18. Cani PD, Delzenne NM. The role of the gut microbiota in energy metabolism and metabolic disease. Curr Pharm Des. 2009;15(13):1546-58.

19. Wu X, Ma C, Han L, Nawaz M, Gao F, Xu Z, et al. Molecular characterisation of the faecal microbiota in patients with type II diabetes. Curr Microbiol. 2010;61:69-78

20. Creely SJ, Mcternan PG, Kusminski CM, Fisher FM, Da Silva NF, Khanolkar M, et al. Lipopolysaccharide activates an innate immune system response in human adipose tissue in obesity and type 2 diabetes. Am J Physiol Endocrinol Metab. 2007;292:740-7.

21. Mahowald MA, Rey FE, Seedorf H, Turnbaugh PJ, Fulton RS, Wollam A, et al. Characterizing a model human gut microbiota composed of members of its two dominant bacterial. Proc Natl Acad Sci U S A. 2009;106:5859-64.

22. Cani PD, Neyrinck AM, Fava F, Knauf C, Burcelin RG, Tuohy KM, et al. Selective increases of bifidobacteria in gut microflora improve high-fat-dietinduced diabetes in mice through a mechanism associated with endotoxaemia. Diabetologia. 2007;50:2374-83.

23. Abbud W, Habinowski S, Zhang JZ, Kendrew J, Elkairi FS, Kemp BE, et al. Stimulation of AMP activated protein kinase (AMPK) is associated with enhancement of Glut1-mediated glucose transport. Arch Biochem Biophys. 2000;380:347.
24. Kong WJ, Zhang H, Song DQ, Xue R, Zhao W, Wei J, et al. Berberine reduces insulin resistance through protein kinase C-dependent up-regulation of insulin receptor expression. Metabolism. 2009;58:109.

25. Yin J, Xing $H$, Ye J. Efficacy of berberine in patients with type 2 diabetes mellitus. Metabolism. 2008;57:712

26. Dong H, Wang L, Lu Z. Berberine in the treatment of type 2 diabetes mellitus: a systemic review and meta-analysis. Evid Based Complement Alternat Med. 2012;2012:591654.

\section{Submit your next manuscript to BioMed Central and we will help you at every step:}

- We accept pre-submission inquiries

- Our selector tool helps you to find the most relevant journal

- We provide round the clock customer support

- Convenient online submission

- Thorough peer review

- Inclusion in PubMed and all major indexing services

- Maximum visibility for your research

Submit your manuscript at www.biomedcentral.com/submit
Biomed Central 